\title{
Análise da atuação dos stakeholders no processo de implementação do Ponto de Cultura Jacquard no município de Jaguarão-RS
}

\author{
Analysis of the stakeholders performance on the implementation process of \\ the Jacquard Point of Culture in the city of Jaguarão-RS
}

\section{Análisis de la actuación de las partes interesadas no proceso de implementación del punto de cultura Jacquard no municipio de Jaguarão-} $R S$

Eduardo Garcia Souza ${ }^{1}$

Icaro Vasques Inchauspe ${ }^{2}$

\begin{abstract}
Resumo
O presente artigo compreende uma análise da atuação das partes interessadas no processo de implementação de um Ponto de Cultura (desdobramento da Política Nacional de Cultura Viva), na cidade de Jaguarão, situada no extremo sul do Rio Grande do Sul. O Ponto de Cultura Jacquard, composto pela Associação de Artesãos local, desponta como uma importante ferramenta para a valorização da cultura local no município e promoção de atividades culturais na cidade. Através desta pesquisa, foi possível analisar o papel de alguns stakeholders identificados como importantes no processo de inserção dessa ação. Por meio de uma abordagem "multilocal", com a participação em reuniões e assembleias, observação do dia-a-dia do funcionamento do Ponto de Cultura, bem como entrevistas com membros das diferentes organizações, identificamos o papel desses stakeholders no cenário local. A Universidade apresenta uma contribuição indireta na formação de profissionais qualificados, ao passo que a Organização Civil de Interesse Público no município foi peça chave para o auxílio técnico na submissão do projeto à Administração Pública, bem como o Centro de Economia Solidária, fornecedor de infraestrutura.
\end{abstract}

Palavras-Chave: Associação dos Artesãos; Jacquard; Ponto de cultura; Stakeholders;

\section{Resumen}

Este artículo incluye un análisis de la función de los interesados en el proceso de implementación de un Punto de Cultura (rama de la Vida Política Nacional de Cultura) en la ciudad de Yaguarón, que se encuentra en el sur de Rio Grande do Sul. El Punto de Cultura Jacquard, compuesto por la Asociación de artesanos locales, se está convirtiendo en una herramienta importante para el desarrollo de la cultura local y la promoción de actividades

\footnotetext{
1 Mestrando em Desenvolvimento Territorial e Sistemas Agroindustriais; Universidade Federal de Pelotas; Pelotas, Rio Grande do Sul - Brasil; admeduardogarcia@gmail.com.

${ }^{2}$ Mestrando em Antropologia; Universidade Federal de Pelotas; Pelotas, Rio Grande do Sul - Brasil; icarovasques@gmail.com.
} 
culturales en la ciudad. A través de esta investigación, se podría analizar el papel de algunos grupos de interés identificados como importantes en el proceso de integración de esta acción. A través de un enfoque "multilocal", con la participación en reuniones y asambleas, la observación de la operación del día a día de los Puntos de Cultura, así como entrevistas con miembros de diferentes organizaciones, identificamos el papel de estos actores en la escena local. La Universidad tiene una contribución indirecta a la formación de profesionales cualificados, mientras que la Organización Civil de Interés Público en la ciudad jugó un papel clave para la asistencia técnica en la presentación del proyecto a la Administración Pública y el Centro de Economía Solidaria, proveedor de infraestructura.

Palabras clave: Asociación de Artesanos; jacquard; punto de cultivo; las partes interesadas;

\begin{abstract}
This article includes an analysis of the performance of stakeholders in the process of implementing a Culture Point (development of the National Policy for Live Culture) in the city of Jaguarão, located in the southernmost part of Rio Grande do Sul. The Jacquard Culture Point, composed by the local Artisans Association, stands out as an important tool for enhancing the local culture in the municipality and promoting cultural activities in the city. Through this research, it was possible to analyze the role of some stakeholders identified as important in the process of insertion of this action. Through a "multilocal" approach, with participation in meetings and assemblies, day-to-day observation of the operation of the Culture Point, as well as interviews with members of different organizations, we identified the role of these stakeholders in the local scenario. The University presents an indirect contribution in the training of qualified professionals, while the Civil Organization of Public Interest in the municipality was a key part for the technical assistance in the submission of the project to the Public Administration, as well as the Solidary Economy Center, an infrastructure provider.
\end{abstract}

Keywords: Association of Artisans; Jacquard; Culture point; Stakeholders;

\title{
1. Introdução
}

O presente artigo é fruto de nossa pesquisa anterior intitulada: "UM OUTRO PONTO DE VISTA: O Ponto de Cultura como ferramenta para o desenvolvimento e preservação das identidades culturais" (GARCIA SOUZA; INCHAUSPE. 2016) publicada na Revista Poder \& Cultura. Nela, havíamos feito uma análise sobre a implantação do Ponto de Cultura Jacquard em Jaguarão-RS, procurando demonstrar como essa ferramenta da política cultural poderia influenciar no desenvolvimento local e no acesso a melhores condições de vida por parte dos atores sociais envolvidos. Limitamo-nos a levantar algumas questões em tom propositivo, haja vista o caráter recente da iniciativa, não foi possível ainda delimitar com clareza os reais reflexos dessa ação na localidade. Os elementos levantados tratam-se obviamente de proposições que com o desdobramento e evolução do Ponto de Cultura Jacquard podem ser melhor analisados em estudos futuros já munidos de práticas resultantes da política implementada.

Neste espaço aprofundamos a discussão iniciada anteriormente e prosseguimos na análise sobre os Pontos de Cultura, desdobramentos da Política Nacional de Cultura Viva (PNCV), instituída pela Lei $\mathrm{n}^{\mathrm{o}} 13.018$, de 22/07/2014, regulamentada pela Instrução Normativa/MinC $n^{\circ}$ 01, de 07/04/2015, que transforma os Pontos e Pontões de Cultura em Política de Estado. 
Precisamente nossa análise continua sobre a avalição da implementação do Ponto de Cultura Jacquard na Associação dos Artesãos de Jaguarão-RS. O acompanhamento durante a implementação possibilitou algumas inferências que são explanadas nas páginas seguintes. A organização estudada é composta por cerca de 30 artesãs que dominam as técnicas de fabricação de artesanato em lã. É importante salientar que as artesãs da Associação procuram manter preservadas os saberes-fazer locais como forma de obter renda que lhes possibilita acesso a condições dignas de vida, no entanto a implementação do ponto de cultura se deu através da interação de diversos atores (stakeholders) e não somente através da própria organização dos trabalhadores da associação.

Dessa forma, nesse espaço procuramos responder qual foi o papel de alguns stakeholders envolvidos no processo de implementação dessa política pública e qual a sua importância para a efetivação do ponto de cultura, tomando o pressuposto de nosso trabalho anterior, de que para ocorrer um desenvolvimento que parte do território, é necessária a atuação de agentes externos.

Este artigo desdobra-se ainda, além da breve introdução ao debate, em dos capítulos que discutem sequencialmente: cultura e política cultural dos pontos de cultura e as partes interessadas (stakeholders) nas políticas públicas. Na sequência são apresentados aspectos metodológicos, análise de dados e por fim, algumas considerações finais.

\section{Cultura e Política Cultural dos Pontos de Cultura}

Partindo do conceito de cultura no viés antropológico, entende-se que não cabe mais a distinção restrita de alta e baixa cultura, típica da visão que separa os cultos dos incultos. A cultura não está circunscrita a círculos, camadas ou grupos sociais: “a cultura pode existir sem consciência de identidade, ao passo que as estratégias de identidade podem manipular e até modificar uma cultura" (CUCHE, 1999, p.177). Cabe ressaltar que cultura é um conceito demasiadamente amplo e não fixo para definir unanimemente.

A partir da ampliação ou até em certos pontos cisão promovida, principalmente no âmbito antropológico, do conceito de alta e baixa cultura, que encontramos indícios mais claros de uma concepção de cultura como um conjunto de saberes-fazeres humano. Podemos defender por este prisma antropológico, que "uma cultura fornece os conhecimentos, valores, símbolos que orientam as vidas humanas" (MORIN, 2003, p. 48).

Destaca-se ainda em Morin, a postura propositiva de como lidar com essa concepção: "A cultura das humanidades foi, e ainda é, para uma elite, mas de agora em diante deverá ser, para todos, uma preparação para a vida” (MORIN, 2003, p.48). Esta leitura de como opera a 

e-ISSN 2016/Atual: 2525-7870 | e-ISSN 2015/2016: 2447-018X

cultura, ou quais aspectos da vida em sociedade ela influencia, aproxima-se cada vez mais da concepção da cultura como processo. Nesse sentindo, a partir do papel do Estado, enquanto produtor por excelência, de políticas públicas (SOUZA, 2006, p.3) em sua forma de governo a partir do ano de 2003, na gestão Gilberto Gil a frente do Ministério da Cultura (MinC) onde há um giro epistemológico de atuação, digamos assim, fazendo reverberar a noção antropológica de cultura na execução das políticas culturais. Segundo Gil:

[...] toda política cultural faz parte da cultura política de uma sociedade e de um povo, num determinado momento de sua existência. No sentido de que toda política cultural não pode deixar nunca de expressar aspectos essenciais da cultura desse mesmo povo. Mas, também, no sentido de que é preciso intervir. Não segundo a cartilha do velho modelo estatizante, mas para clarear caminhos, abrir clareiras, estimular, abrigar. Para fazer uma espécie de "do-in" antropológico, massageando pontos vitais, mas momentaneamente desprezados ou adormecidos, do corpo cultural do país... (GIL, 2003, p. 3)

Com isso, Rubim (2007) demonstra que ao adotar a concepção antropológica de cultura, o governo estatal ampliou a abrangência das políticas culturais para o âmbito das culturas populares, afro-brasileiras, indígenas, das periferias, entre outras.

Botelho (2001) aponta que este processo determinante de escolha política e cultural ocorre através da interação social dos indivíduos, que elaboram seus modos de pensar e sentir, constroem seus valores, manejam suas identidades e diferenças e estabelecem suas rotinas. Desta forma, cada indivíduo ergue à sua volta, e em função de determinações de tipo diverso, pequenos mundos de sentido que lhe permitem uma relativa estabilidade. Sob esta perspectiva, a proposta de cidadania cultural concebe a cultura como direito de todos os cidadãos e o Estado como agente da política cultural.

Para tanto, a partir do ano de 2012, por meio da Emenda Constitucional $\mathrm{n}^{\mathrm{o}} 71 / 2012$, do Ministério da Cultura (MinC), foi aprovado o Sistema Nacional de Cultura (SNC), que tem como um de seus objetivos a adesão dos municípios ao SNC em todo o território.

Dando sequência a política cultural supracitada, no ano de 2014, ocorreu o edital de chamamento e seleção de entidades para o desenvolvimento do projeto "Rede RS Pontos de Cultura do Programa Cultura Viva no Estado do RS" por intermédio da Secretária de Estado da Cultura (SEDAC) $)^{3}$ do Programa Cultura Viva do Ministério da Cultura.

A Associação dos Artesãos de Jaguarão através de suas atividades já desenvolvidas em artesanato em lã, foi classificada em $1^{\circ}$ lugar geral com a proposta de tornar-se Ponto de

\footnotetext{
${ }^{3}$ Atualmente a SEDAC passou a chamar-se SEDACTEL (Secretária da Cultura, Turismo, Esporte e Lazer) devido a reforma na administração pública do Estado do Rio Grande do Sul no ano de 2016.
} 
Cultura Jacquard - O objetivo do Ponto, é o desenvolvimento de atividades de formação artística e de troca de saber e conhecimento com o intuito de preservar a memória, história e salvaguardar a técnica do Jacquard praticada pelos membros da Associação dos Artesões de Jaguarão. Estão previstas ações, tais como: oficinas de Crochê Jacquard, tricô, oficina de tear, em um processo que vai desde a extração da matéria prima, a lavagem da lã, o cardeamento, a fiação em roca ou em fuso e finalizando com a confecção de peças diversificadas, além de rodas de memória e conversas.

Mas afinal, o que são Pontos de Cultura e como podem ser entendidas sob o aspecto de sua funcionalidade como uma política pública?

Compreende-se que os Pontos de Cultura são elos entre a sociedade e o Estado que possibilitam o desenvolvimento de ações culturais sustentadas pelos princípios da autonomia, protagonismo e empoderamento social, integrando uma gestão compartilhada e transformadora da instituição selecionada com a Rede de Pontos de Cultura.

Neste sentido, o Ponto de Cultura funcionará como um instrumento de pulsão e articulação de ações e projetos já existentes nas comunidades do Estado do Rio Grande do Sul, desenvolvendo ações continuadas em pelo menos uma das áreas de Culturas Populares, Grupos Étnico-Culturais, Patrimônio Material e Imaterial, Audiovisual e Radiodifusão, Culturas Digitais, Gestão e Formação Cultural, Pensamento e Memória, Expressões Artísticas, e/ou Ações Transversais.

A partir do entendimento da Associação dos Artesãos já estarem incluídas sob uma cultura tradicional ou cultura popular, e a partir de seu objeto de trabalho, sendo um patrimônio material histórico, imaterial humano (saberes-fazeres e memórias vivenciadas) das associadas, foi onde ocorreu o insight para elaboração do projeto e implementação da atividade, intercalada entre os stakeholders, tornando-se um grupo coerente em torno de objetivos pessoais e profissionais.

Este tipo de política pública com caráter distributivo ${ }^{4}$ e real, proporcionou o encontro de agentes e instituições propiciando o encontro de ideias e sua organização, análise e viabilidade de implementação das ações, captação e avaliação dos insumos necessários, e uniformidade do projeto até a tomada de decisão para sua implementação.

Para verificar estes resultados, só será possível a partir de um período constante das atividades da Associação dos Artesãos, da qual será necessário fazermos a análise e impacto

\footnotetext{
${ }^{4}$ Sobre o conceito de distributivo ver (LOWI, 1972; SECCHI, 2015).
} 
dos resultados implementados. Futuramente, em momento oportuno, os autores pretendem fazer tais análises.

\section{Políticas Públicas e stakeholders}

Conforme Souza (2006) as políticas públicas apresentam-se como importante área de conhecimento, elucidando regras e modelos que regem sua decisão, elaboração, avaliação e implementação (caso do presente estudo). No entanto, as múltiplas formas de interpretar o contexto de implementação da política pública requerem, que esta, esteja investida de multidisciplinaridade. Assim, neste capítulo procuramos trazer luz à discussão da atuação dos agentes que se envolvem na implementação de uma política pública.

A implantação de determinada política pública está inerente à um contexto conflituoso, dentre as mais diversas concepções ideológicas daqueles que atuam na linha de frente dessa prática. Assim, uma política pública pode ser entendida como:

Fundamentalmente, uma política pública é interpretada aqui como um conjunto de ideias institucionalizadas, o marco analítico proposto visando reconstruir e seguir a trajetória de ideias que levem à sua institucionalização, quer dizer à sua transformação ("concretização") em instrumentos das políticas públicas. Ao descrever o caminho das ideias desde o lócus de sua produção até a sua institucionalização, o objetivo consiste em salientar que tal trajetória não é apenas contínua, mas também corresponde a um processo específico de seleção. Em todo este processo, as políticas públicas emergem, são mantidas ou rejeitadas, apenas um pequeno número delas sendo finalmente discutido nas mesas formais de negociação (FOUILLEUX, 2011, p. 93).

No entanto, não cabe aqui, voltar ao plano da origem da ferramenta analisada para compreender ontologicamente sua concepção por meio de campo de disputa de ideias. O que se procura é discutir a forma com que uma vez munidos da política pública, os agentes locais responsáveis por sua implementação agem.

Segundo Rua (1997) a característica principal da sociedade moderna são as diferenciações de ideias, valores, interesses e aspirações entre os atores sociais, o que leva a conflito entre esses atributos (valores, interesses e opiniões). Como Foullieux (2011) nos conta, essa questão está intimamente relacionada aos atores políticos ou stakehoders, uma vez que estão defendendo seus interesses quando decidem apoiar determinada ideia, ação ou corrente política. Analisar as políticas públicas sob a ótica dos interesses, significa identificar os stakeholders envolvidos no processo, as estratégias desenvolvidas por esses atores (custo benefício de tomar determinada posição em função de um resultado esperado) e a lógica que permeia a ação coletiva (TECCHIO et al.,2015). 

e-ISSN 2016/Atual: 2525-7870 | e-ISSN 2015/2016: 2447-018X

A identificação dos atores, portando é parte fundamental do processo de análise de políticas públicas. Rua (1997) destaca que dentre os critérios existentes para identificar os atores envolvidos em uma política pública, o mais eficaz e simplista é o estabelecimento daqueles que tem alguma "coisa" em jogo na questão, isto é, identificar quem são as pessoas que podem ganhar ou perder com determinada política. $\mathrm{O}$ pressuposto aceito de que os atores envolvidos nas questões políticas agem de forma a atender seus interesses, todavia não significa como destacam Tecchio et al. (2015) que eles agem buscando otimizar seus resultados e como destaca as autoras, para análise da política pública é importante compreender os interesses que fazem com que determinados grupos sociais participem das políticas públicas.

Assim, demonstramos a atuação dos stakeholders no processo de implantação do ponto de cultura no município de Jaguarão e a identificação de seu interesse nesse evento.

\section{Aspectos metodológicos}

Primeiramente procurou-se mapear o papel central das instituições envolvidas no processo de implementação do Ponto de Cultura e de que forma elas se conectam e se associam em sua implementação efetiva. A metodologia aplicada, se deu basicamente no trabalho de campo por meio da observação participante em algumas reuniões diretamente com a Associação dos Artesãos, entrevistas com agentes culturais, e indivíduos que atuam em Instituições Locais. A metodologia aplicada ocorreu de forma multisituada ou "multillocal" (Marcus, 2001) prevendo maior abrangência possível sobre o objeto de estudo abordado, onde procurou-se desenvolver em múltiplos cenários de atividade entre os atores participantes no contexto de efetivação do Ponto de Cultura.

Nesse sentido, para descobrimento das instituições analisadas, traçamos os seguintes parâmetros metodológicos a partir da abordagem pautada em dois passos cruciais para a análise. 1) A participação da realidade vivenciada no local, através das reuniões e movimentos do grupo em particular - Associação dos Artesãos, agentes culturais da OSCIP e Instituições Locais. 2) Compreender de forma subjetiva a interação das instituições e seu papel na composição da política pública implementada, através de conversas com as partes interessadas que de alguma forma tiveram atuação na concepção desse evento, ainda que de forma parcial ou indireta.

Delimitamos geograficamente a região de estudo na cidade de Jaguarão-RS. Compreendendo em sua amostra, os atores sociais que fazem parte da Associação dos Artesãos, bem como a Organização da sociedade civil de interesse público responsável pela 

e-ISSN 2016/Atual: 2525-7870 | e-ISSN 2015/2016: 2447-018X

implementação do "Ponto de Cultura Jacquard", identificada aqui como OSCIP X, a Universidade local e o Centro de Economia Solidária do município, participantes do processo de implantação. Abaixo apresenta-se o quadro 1 com o resumo dos stakeholders

\begin{tabular}{rrl}
\hline Stakeholders & $\begin{array}{r}\text { Membros } \\
\text { entrevistados }\end{array}$ & Método \\
OSCIP X & Voluntário & Entrevista \\
Associação dos \\
Artesãos & Artesãs & Entrevista/Observação \\
Universidade & Professores/E & Entrevista \\
studantes & \\
Centro de & Funcionário & Observação \\
\hline
\end{tabular}

Quadro 1 - Descrição das instituições analisadas

Fonte: Elaborado pelos autores

\section{Análise}

Na região de Jaguarão, a presença intensa de uma tradição, a qual é o artesanato em lã, permite que através de características do próprio território, seja levada a cabo uma promoção de condições de vida, muito em ocasião da existência de uma política pública voltada para atender a organização em questão, identificada como o "Ponto de Cultura Jacquard", que é pautado por maioria de suas atividades artesanais, contendo a finalidade artística e utilitária. Procuramos aqui discutir o papel das partes interessadas que fizeram parte do processo de implementação do ponto de cultura no município.

O papel em geral dos stakeholders analisados tipifica uma variedade em suas administrações pública em nível federal/local (Universidade - Centro de Economia Solidaria) 

e-ISSN 2016/Atual: 2525-7870 | e-ISSN 2015/2016: 2447-018X

juntamente com organizações sociais de interesse público (Associação dos Artesãos e OSCIP $\mathrm{X})$. Mas de que forma elas se complementam e se solidarizaram na efetivação do Ponto de Cultura Jacquard?

\subsection{O papel da Universidade}

A importância da Universidade local nesse processo, não se deu diretamente, através de projetos voltados para a construção do ponto de cultura. No entanto a disponibilidade do curso de graduação em Produção Política e Cultural foi de vital importância para o desencadeamento desse processo. A OSCIP X, organização analisada a seguir, é formada por membros egressos desse curso oferecido em nível de bacharelado. Nesse sentido, o papel da Universidade, foi de provedor de formação profissional, através de uma capacitação específica que ensejou o desdobramento futuro de ações voltadas para o fortalecimento da cultura local.

Assim, percebe-se que é imprescindível a cooperação e atuação de uma Universidade pública, gratuita e de qualidade que dissemine e integre o conhecimento em suas atividades de ensino, no processo de aprendizagem e cognição do processo educacional, por meio da pesquisa, através do conhecimento tácito, seja experimental ou não, e indagador por meio do descobrimento de novas possibilidades de aprendizado e seus campos multidisciplinares, e finalizando por meio das ações de extensões que são efetivamente por excelência a sociabilização entre universidade (instituição) e a comunidade em geral (indivíduos).

\subsection{A OSCIP X}

A Organização Social de Interesse Pública, título concebido pelo Ministério da Justiça do Brasil, tem a finalidade de facilitar o aparecimento de parcerias e convênios com todos os níveis de governo e órgãos públicos. Nesse sentido, dentro do Ponto de Cultura, atuou como um intermediador de interesses privados sob uma tutela (financiamento) do setor público.

A partir do conhecimento tecnocrático na administração pública conseguiu dar o suporte necessário para elaboração técnica do projeto e demais competências burocráticas. Sobressaindo, como agente que exerceu forte impacto nas ações efetivas necessárias para a concretização desse processo. Os agentes da organização tiveram papel fundamental para atuar de maneira coordenada com a Associação dos Artesãos, compreendendo suas demandas sociais e traduzindo-as frente ao interesse da Administração Pública. 


\subsection{Centro de Economia Solidária}

Todo esforço despedindo, só foi capaz de desdobrar-se em ações efetivas a partir de um Centro de Economia Solidaria, onde podem idealizar novas formas de convivência e de organização, amparadas na cooperação, na equidade, na sustentabilidade e na solidariedade, e vão na contramão do modelo hegemônico de produção.

O Centro de Economia Solidária do município é parte integrante e fundamental desse processo, uma vez que forneceu toda a infraestrutura necessária, tanto para a realização de cursos, quanto para o estabelecimento de um local para a venda dos artesanatos produzidos pelas artesãs. Além disso, o centro disponibiliza local para a produção e reunião dos membros da associação, que enxergam nele um espaço de convivência entre pares, mas também um forte elo na promoção dos seus produtos e na disseminação de sua cultura para a comunidade como um todo.

\subsection{Associação dos Artesãos}

A Associação dos Artesãos surge no ano de 2004, composta inicialmente por um grupo de 20 mulheres (associadas) que viram a oportunidade de se organizar, para produzir e vender seus artesanatos. Primeiramente o grupo era composto por mulheres da terceira idade, muitas das quais já praticavam as técnicas de artesanato, repassados através das gerações. Os produtos são feitos a partir da esquila da lã das ovelhas da região, uma importante atividade econômica rural do município de Jaguarão (GARCIA SOUZA; INCHAUSPE, 2016),

É uma instituição privada com finalidade pública, e em suma, as maiorias das atividades da organização são desenvolvidas em torno da lã. Sua atuação diante da implantação do ponto de cultura, foi como agente idealizador. No Quadro 2, demonstra-se o resumo do papel dos principais stakeholders identificados na análise.

\begin{tabular}{rrr}
\hline Stakeholders & Atuação & $\begin{array}{r}\text { Importância na } \\
\text { participação }\end{array}$ \\
\hline OSCIP X & $\begin{array}{c}\text { Elaboração e } \\
\end{array}$ & Alta \\
& submissão do projeto à &
\end{tabular}

Associação de

Idealizadora

Alta

Artesãos 
RELACult - Revista Latino-Americana de Estudos em Cultura e Sociedade

Revista Latinoamericana de Estudios en Cultura y Sociedad | Latin American Journal of Studies in Culture and Society V. 03, ed. especial, ago., 2017, p. 154-165 | relacult.claec.org e-ISSN 2016/Atual: 2525-7870 | e-ISSN 2015/2016: 2447-018X

Universidade

Qualificação

Alta

profissional

Centro de

Fornecimento de

Alta

Economia Solidária

Infraestrutura

Quadro 2 - Síntese da atuação dos stakeholders

Fonte: elaborado pelos autores

\section{Considerações finais}

É evidente que, a implantação do ponto de cultura, parte do pressuposto de que a promoção das atividades de artesanato em lã realizadas em Jaguarão pode contribuir para o desenvolvimento local parte do entendimento de Renato Ortiz (2008), onde explica que a existência de um vínculo entre cultura e desenvolvimento é determinante, uma vez que "permite trabalhar temas como: erradicação da pobreza, melhoria das condições de gênero, incentivo ao turismo, preservação do meio ambiente" (ORTIZ, 2008, p. 126).

Nesse sentido, fala-se por meio do incremento nas condições de vida da população local. No entanto, a consolidação desses processos de desenvolvimento não é simples, dependem de uma emancipação dos atores e do reconhecimento de suas habilidades, pois assim, se consegue aflorar a capacidade dos atores locais promover o desenvolvimento através de características endógenas. O desenvolvimento endógeno, não acontece de modo óbvio num processo natural por meio dos atores locais, é imperativa a participação de agentes e instituições de fora do território, visto que esse desenvolvimento crê na influência mútua entre forças locais e de fora da localidade, ou seja, forças endógenas e exógenas.

\section{Referências}

BOTELHO, Isaura. Dimensões da cultura e políticas públicas. São Paulo em perspectiva, v. 15, n. 2, 2001.

CUCHE, D. A Noção de cultura nas ciências sociais. Bauru: EDUSC, 1999.

FOUILLEUX, E. Analisar a mudança: políticas públicas e debates num sistema em diferentes níveis de governança. Estudos sociedade e agricultura, v. 2, 2013.

GIL, G. Discursos do Ministro da Cultura Gilberto Gil. Brasília: Ministério da Cultura, 2003. 

e-ISSN 2016/Atual: 2525-7870 | e-ISSN 2015/2016: 2447-018X

LOWI, T. Four System of Policy: Politics, and Choice. Public Administration Review, v.32, n.4, p.298-310, 1972.

MARCUS, G. Etnografía en/del sistema mundo. El surgimiento de la etnografía multilocal. Revista Alteridades, vol. 11, núm. 22, julio-diciembre, 2001, pp. 111-127. Universidad Autónoma Metropolitana Unidad Iztapalapa Distrito Federal, México. 2001.

MORIN, E. A cabeça bem-feita: repensar a reforma, reformar o pensamento. $8^{\mathrm{a}}$ ed. Rio de Janeiro: Bertrand Brasil, 2003. O Método 1 - A natureza da natureza. Porto Alegre: Sulina, 2008.

PRESIDENCIA DA REPÚBLICA. LEI $N^{o} 13.018$, de 22 de julho de 2014, que institui a Política Nacional Cultura Viva. Disponível em:

<http://www.planalto.gov.br/ccivil_03/_ato2011-2014/2014/lei/113018.htm.>. Acessado em $31 / 03 / 17$.

RUA, M. G. Análise de políticas públicas: conceitos básicos. Manuscrito, elaborado para el Programa de Apoyo a la Gerencia Social en Brasil. Banco Interamericano de Desarrollo: INDES, p. 1-19, 1997.

RUBIM, L. Organização e Produção da Cultura. Salvador. EDUFBA. 2005.

SECCHI, L. Políticas Públicas: Conceitos, Esquemas de Análise, Casos Práticos. São Paulo: Cengage Learning, 2011. 133p.

SOUZA, C. Políticas públicas: uma revisão da literatura. Sociologias, Porto Alegre, v. 8, n. 16, p. 20-45, 2006.

SOUZA, E. G.; INCHAUSPE, I. V. Um outro ponto de vista: O Ponto de Cultura como ferramenta para o desenvolvimento e preservação das identidades culturais. Poder e Cultura, v. 3, p. 245-262, 2016.

TECCHIO, A., Grisa, C., Oliveira, L. Z., Cazella, A. A. Ideas, intereses e instituciones en la política de desarrollo territorial brasileña: un estudio en el Território Meio Oeste Contestado. Quito: Revista de Ciencias Sociales, n. 53, p. 105-125 set/ 2015. 